\title{
Small-Angle X-Ray Scattering from Semidilute Polymer Solutions. Deuterated Polystyrene-Deuterated Cyclohexane System
}

\author{
Takashi ICHIMURA, Koji OKANO, ${ }^{*}$ Kimio KURITA, \\ and Eiichi WADA \\ College of Science and Technology, Nihon University, \\ Kanda Surugadai, Chiyoda-ku, Tokyo 101, Japan \\ *Department of Applied Physics, Faculty of Engineering, \\ The University of Tokyo, Hongo, Bunkyo-ku, \\ Tokyo 113, Japan
}

(Received November 7, 1987)

\begin{abstract}
Binary $\left(B_{1}\right)$ and ternary $\left(B_{2}\right)$ cluster integrals of deuterated polystyrene in deuterated cyclohexane were determined by small-angle $\mathrm{X}$-ray scattering from semidilute solutions. The results were compared with those previously determined for the undeuterated polystyrenedeuterated cyclohexane system and undeuterated polystyrene-undeuterated cyclohexane system. It was found that for all cases $B_{2}$ was unaltered by deuterium substitution within the experimental accuracy and had the value $0.8 \times 10^{-51} \mathrm{~cm}^{6}$ almost independently of temperature, whereas $B_{1}$ had the temperature dependence of the form $B_{1}=B_{10}(1-\theta / T)$ near the $\theta$ temperature, where temperature independent factor $B_{10}$ was also unaffected by deuteration; the sole deuteration effect observed was a shift of $\theta$ temperature at which $B_{1}$ vanishes.

KEY WORDS Deuteration Effect / Semidilute Solutions / Deuterated

Polystyrene / Deuterated Cyclohexane / Small-Angle X-Ray Scattering /
\end{abstract}

In a previously published work $^{1}$ we measured the interaction parameters of polystyrene (PSH) in deuterated cyclohexane $\left(\mathrm{C}_{6} \mathrm{D}_{12}\right)$ by small-angle neutron scattering (SANS) from semidilute solutions. We found that the binary $\left(B_{1}\right)$ and ternary $\left(B_{2}\right)$ cluster integrals of polymer segment in solution have the following temperature dependence near the $\theta$ temperature at which $B_{1}$ vanishes:

$$
\begin{aligned}
& B_{1}=B_{10}(1-\theta / T) \\
& B_{10}=6 \times 10^{-27} \mathrm{~cm}^{3} \\
& B_{2}=0.8 \times 10^{-51} \mathrm{~cm}^{6}
\end{aligned}
$$

where $\theta$ was $40^{\circ} \mathrm{C}$.

A later small-angle X-ray scattering (SAXS) measurement ${ }^{2}$ of semidilute solutions of undeuterated polystyrene (PSH) in undeuterated cyclohexane $\left(\mathrm{C}_{6} \mathrm{H}_{12}\right)$ revealed that the tem- perature dependences of the interaction parameters are the same as those of (1), (2), and (3) for PSH- $\mathrm{C}_{6} \mathrm{D}_{12}$ system except for the fact that $\theta$ in this case was $35^{\circ} \mathrm{C}$.

We also demonstrated that these interaction parameters explain quantitatively the difference between the coexistence curves of PSH$\mathrm{C}_{6} \mathrm{H}_{12}$ and $\mathrm{PSH}-\mathrm{C}_{6} \mathrm{D}_{12}$ systems ${ }^{3}$.

The purpose of the present paper is to study further the deuteration effect on interaction parameters of polystyrene-cyclohexane system we measure SAXS from semidilute solutions of deuterated polystyrene (PSD) in $\mathrm{C}_{6} \mathrm{D}_{12}$, from which we extracted the temperature dependences of $B_{1}$ and $B_{2}$ near $\theta$-point. In the SAXS MEASUREMENT we describe our experimental procedure and results. In DISCUSSION, we discuss briefly the implications of the results. 


\section{SAXS MEASUREMENT}

We consider semidilute polymer solutions in the poor solvent regime the condition of which was discussed in detail previously. ${ }^{4}$ The angular dependence of the intensity $I(q)$ of the scattered $\mathrm{X}$-rays is given by the following equations $^{5}$ in the (so-called) intermediate range in which scattering vector $q$ lies between the reciprocal of the radius of gyration of a polymer chain and that of the size of a polymer segment:

$$
I(q)^{-1}=I(0)^{-1}\left(1+\xi^{2} q^{2}\right)
$$

where the correlation length $\xi$ is given by

$$
\left(\frac{1}{\xi^{2}}-\frac{6}{M A^{2}}\right) / C=12 N_{\mathrm{A}} \frac{B_{1}}{A^{2}}+36 N_{\mathrm{A}}{ }^{2} \frac{B_{2}}{A^{2}} C
$$

In the above equations, $M$ is the molecular weight of a polymer $\left(M A^{2} / 6\right)^{1 / 2}$ is the unperturbed gyration radius of a polymer molecule, $N_{\mathrm{A}}$ the Avogadro's number. As discussed previously, ${ }^{2} \mathrm{~A}$ is practically constant in the measured temperature range and equals to $0.7 \AA$.

SAXS measurements were carried out by using a Kratky U-slit camera with entrance slit of $100 \mu \mathrm{m}$, counter slit of $300 \mu \mathrm{m}$, and height-determining slit of $16 \mathrm{~mm}$. A RigakuDenki RU-200 rotating copper anode X-ray generator was used as $50 \mathrm{kV}, 200 \mathrm{~mA}$ and a wavelength $\lambda=1.54 \AA$ was selected with $\mathrm{Ni}$ filter of $14 \mu \mathrm{m}$ thickness. Photon counting was done by a scintillation counter with pulseheight analyser. Slit correction was done by the variation-function method. ${ }^{6}$ The sample cell used was $1.97 \mathrm{~mm}$ thickness.

Samples used were solutions of monodisperse deuterated polystyrene (from Polymer Laboratories Co.; 99.2 atom \% deuterated; molecular weight $\left.5 \times 10^{5} ; \bar{M}_{w} / \bar{M}_{n}<1.05\right)$ in deuterated cyclohexane $(99.5$ atom $\%$ deuterated). The temperature of the sample solution was controlled within $\pm 0.03^{\circ} \mathrm{C}$ by using a thermocouple placed in the solution.

As shown in Figure 1 the measured scattered intensity obeyed eq 4 in the range $q^{2}<4 \times$

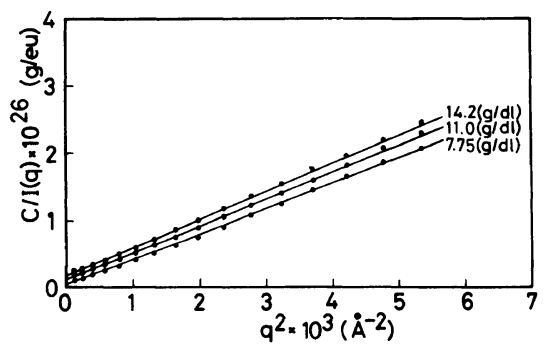

Figure 1. Examples of measured angular dependences of SAXS at $36.0^{\circ} \mathrm{C}$.

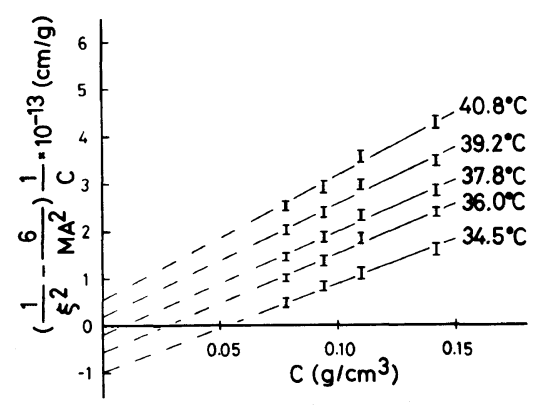

Figure 2. Concentration dependence of $\xi$ for several temperatures near the $\theta$ point.

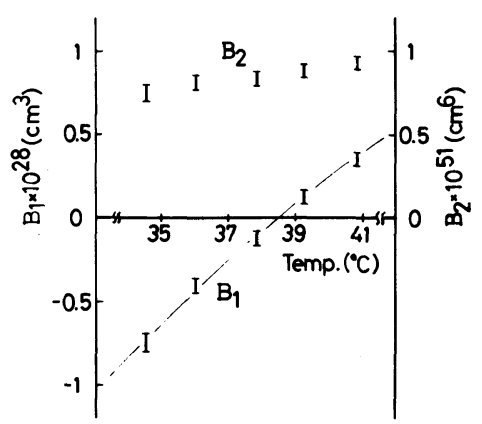

Figure 3. Temperature dependence of binary $\left(B_{1}\right)$ and PSD- $\mathrm{C}_{6} \mathrm{D}_{12}$

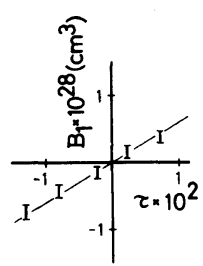

Figure 4. Binary cluster integral $B_{1}$ of PSD- $C_{6} D_{12}$ system plotted against $\tau$. 
$10^{-3} \AA^{-2}$. From this figure we could determine the correlation length $\xi$ for each concentration and temperature. The concentration dependence of $\xi$ for each temperature turned out to agree well with the prediction of eq 5 . Thus we were able to determine $B_{1}$ and $B_{2}$ separately as a function of temperature. Figure 3 shows the temperature dependence of $B_{1}$ and $B_{2}$ thus obtained. We see from this figure that $\theta$ temperature of this system is about $38.5^{\circ} \mathrm{C}$. In Figure 4 the values of $B_{1}$ are replotted against reduced temperature $\tau$ defined by

$$
\tau=1-\theta / T
$$

We thus recognize that the temperature dependences of $B_{1}$ and $B_{2}$ near $\theta$ point are expressed by the same equations as those for PSH $-\mathrm{C}_{6} \mathrm{D}_{12}$ and $\mathrm{PSH}-\mathrm{C}_{6} \mathrm{H}_{12}$, eq 1,2 , and 3 , except that $\theta$ for PSD- $\mathrm{C}_{6} \mathrm{D}_{12}$ system is $38.5^{\circ} \mathrm{C}$.

\section{DISCUSSION}

We conclude from the present experimental results our previous works ${ }^{1,2}$ that the effect of deuterium substitution either of polystyrene or cyclohexane or both on $B_{1}$ and $B_{2}$ is solely the shift of $\theta$ temperature which is defined as the temperature where $B_{1}$ vanishes while $B_{10}$ and $B_{2}$ are kept unchanged within the experimental accuracy.

$\theta$ temperatures as obtained by our SANS and SAXS measurements are listed in Table I together with those obtained by Strazielle and Benoit. ${ }^{7}$ Note that the $\theta$ temperatures of these authors are defined as the temperature where

Table I. $\theta$ temperatures of deuterated and undeuterated polystyrene-cyclohexane systems

\begin{tabular}{ccc}
\hline System & $\begin{array}{c}\theta\left({ }^{\circ} \mathrm{C}\right) \text { from SAXS } \\
\text { or SANS }\end{array}$ & $\theta\left({ }^{\circ} \mathrm{C}\right)$ from $A_{2}$ \\
\hline PSH-C ${ }_{6} \mathrm{H}_{12}$ & 35 & 35 \\
PSH-C $\mathrm{C}_{6} \mathrm{D}_{12}$ & 40 & 40 \\
PSD- ${ }_{6} \mathrm{D}_{12}$ & 38.5 & 36 \\
\hline
\end{tabular}

the second virial coefficient $A_{2}$ vanishes. In spite of the difference in definition and the method of measurement we have almost the same value for each system.

A rough qualitative aspect of the above mentioned results may be explained on the basis of lattice model with van der Waals dispersion interaction. ${ }^{3}$ In this model the interaction parameters are respectively given by

$$
\begin{aligned}
& B_{1}=m^{-1 / 2} A^{3}(1-2 \chi) \\
& B_{2}=A^{6} \\
& \chi=\frac{\kappa}{2 T}\left(\alpha_{\mathrm{p}}-\alpha_{\mathrm{s}}\right)^{2}
\end{aligned}
$$

where $m$ is the molecular weight of a polymer segment, $\alpha_{p}$ and $\alpha_{s}$ are the polarizabilities of a polymer segment and a solvent molecule respectively, and $\kappa$ is a positive constant. In this model $B_{10}$ and $\theta$ are respectively given by

$$
\begin{aligned}
& B_{10}=m^{-1 / 2} A^{3} \\
& \theta=\kappa\left(\alpha_{\mathrm{p}}-\alpha_{\mathrm{s}}\right)^{2}
\end{aligned}
$$

The last equation is consistent with Table I provided that

$\left(\alpha_{p}{ }^{H}-\alpha_{s}{ }^{D}\right)^{2}>\left(\alpha_{p}{ }^{D}-\alpha_{s}{ }^{D}\right)^{2}>\left(\alpha_{p}{ }^{H}-\alpha_{s}{ }^{H}\right)^{2}$

where superscripts $H$ and $D$ denote, respectively, the undeuterated and deuterated objects. In order to go into more detail we need a statistical mechanical theory of solutions based on the detailed knowledge of the bare interaction between solute and solvent molecules. In fact some works along this line have recently been published on the mixture of undeuterated and deuterated polystyrene, but there remains some controversial points. ${ }^{8-10}$ We hope that this kind of calculation with sound physical basis will be done for polymer-solvent systems in the near future.

\section{REFERENCES}

1. K. Okano, K. Kurita, S. Nakajima, E. Wada, M. Furusaka, and Y. Ishikawa, Physica, 120B, 413 (1983). 


\section{T. ICHIMURA et al.}

2. T. Ichimura, K. Okano, K. Kurita, and E. Wada, Polymer, 28, 1573 (1987).

3. T. Ichimura, K. Okano, K. Kurita, and E. Wada, Polym. J., 19, 1101 (1987).

4. K. Okano, T. Ichimura, K. Kurita, and E. Wada, Polymer, 28, 693 (1987).

5. M. A. Moore, J. Phys. (Paris), 38, 265 (1977).

6. O. Glatter, J. Appl. Crystallogr., 7, 147 (1974).
7. C. Strazielle and H. Benoit, Macromolecules, 8, 203 (1975).

8. F. S. Bates and G. D. Wignall, Phys. Rev. Lett., 57, 1429 (1986).

9. R. R. Singh and W. A. Van Hook, J. Chem. Phys., 86, 2969 (1987).

10. R. R. Singh and W. A. Van Hook, Macromolecules, 20, 1855 (1987). 